\title{
Impact des rejets sur les millieux récepteurs
}

\author{
par Elodie Brelot
}

Directeur du GRAIE

\author{
Bernard Chocat
}

Professeur à l'INSA de Lyon

\section{I 圆 LES PHÉNOMENES D'IMPACT}

Le terme d'impact est utilisé pour désigner l'ensemble des conséquences directes ou indirectes des rejets sur :

- le milieu naturel (le biotope);

- les espèces végétales et animales qui y vivent ou en dépendent (la biocénose):

- les activités qui y sont pratiquées ou qui l'utilisent (les usages).

Ce terme générique recouvre différents phénomènes, dépendant eux-mêmes d'un grand nombre d'éléments: diversité des sources de pollution, des polluants euxmêmes, des interactions physiques, chimiques et biologiques entre les différents paramètres et enfin diversité des perceptions de la qualité de l'eau.

\subsection{Définition de l'impact}

Par ses rejets, l'homme introduit dans les milieux récepteurs soit des quantités anormales d'éléments qui y étaient déjà présents, soit des éléments nouveaux. Dans les deux cas, il modifie le fonctionnement de l'écosystème en déséquilibrant sa cinétique. L'impact d'un rejet particulier peut ainsi être défini comme la part spécifique de responsabilité de ce rejet dans la modification du milieu.

Cette modification est généralement perçue comme une dégradation. En réalité, la notion de dégradation reste relative et subjective en ce sens qu'elle fait référence à la qualité des milieux naturels qui constitue elle-même une notion relative et subjective.

On peut identifier deux perceptions différentes de la qualité des milieux naturels, de leur dégradation et donc de l'impact d'un rejet :

- La qualité écologique est relative à l'équilibre général de l'écosystème, et notamment de la biocénose. On considère que le milieu est de bonne qualité si son état est voisin d'un état naturel de référence, souvent difficile à définir.

- La perception environnementaliste de la qualité revient à considérer le milieu comme l'environnement de l'homme. Le milieu naturel doit être adapté aux usages que l'homme veut en faire: fabrication d'eau potable, bai-

L'impact des rejets urbains de temps de pluie est une notion très sotwent discutée qui recouvre une réalité complexe. La première partie de cette communication tente de donner différents éclairages sur la notion d'impact de manière a préciser la défnition de ce terme. Sont ainsi analysés les critères permettant de distinguer les différents types d'impact: échelles de temps et d'espace, compartiments considérés de l'hydrosystème. Une attention particulière est portée sur la distinction entre la perception écologique, qui considère les impacts du point de vue de la préservation du milieu, et la perception environnementaliste qui les considère du point de vue des usages. La contribution spécifique des rejets urbains de temps de pluie est mise en valeur. La dernière partie de la communication traite des divers milieux récepteurs et de leurs sensibilités particulières aux différents impacts recensés. 
gnade, pêche, etc. Le milieu sera donc considéré comme dégradé si sa qualité rend difficile, voire impossible, un usage souhaité.

C'est généralement cette dernière définition, bien que réductrice, qui est considérée à la fois par le grand public et par beaucoup de techniciens.

Nous proposons pour notre part de caractériser l'impact d'un rejet sur un milieu aquatique par la différence entre l'état du milieu perturbé par le rejet et l'état hypothétique du milieu en l'absence du rejet. Sur les cours d'eau, cet état hypothétique peut être défini : soit par référence à l'état du milieu à l'amont du rejet (si celui-ci est permanent), soit par référence à l'état avant le rejet (s'il s'agit d'un rejet événementiel), soit enfin par référence à un état théorique.

Les phénomènes d'impact dépendent de la dynamique des rejets. Les rejets de temps sec et de temps de pluie apportent des éléments dans des proportions variables, mais surtout selon des dynamiques différentes. Les rejets de stations d'épuration en fonctionnement normal sont continus et relativement stables, ils ont donc un effet durable avec des phénomènes d'accumulation probablement plus importants que pour les rejets de temps de pluie. Ces derniers sont événementiels et peuvent parfois être très violents, pouvant de fait provoquer des effets de choc.

La sévérité des phénomènes d'impact est également fonction de la réaction du milieu. L'état à l'amont des rejets, la capacité d'autoépuration et la sensibilité particulière des usages pratiqués ou souhaités sur le milieu sont des éléments intervenant dans les phénomènes d'impact ou déterminant pour juger de leur gravité. La caractérisation de l'impact des rejets nécessite par conséquent une analyse des deux sous-systèmes, bassins d'apport et milieu récepteur, et de leur interface.

Deux types d'impact peuvent être distingués selon leurs dynamiques :

- Les effets de choc, immédiats, ou à court terme, sont caractérisés par une dégradation momentanée du milieu. Le biotope récupère rapidement pour retrouver sa qualité initiale, comme nous le représentons dans la figure l, mais la biocénose peut s'en trouver affectée de façon plus ou moins irrémédiable. Ce sont essentiellement les conséquences de rejets instantanés. [Chocat et al., 1993] proposent de considérer qu'une pollution est à effet de choc si le temps de récupération du milieu est inférieur à l'intervalle moyen séparant deux événements. L'analyse de ces phénomènes nécessite la prise en considération des pics de pollution associée à celle de leur fréquence. On considérera par exemple la masse journalière maximum susceptible d'être rejetée par un événement, en moyenne une fois tous les dix ans.

- Les effets cumulatifs ou différés sont eux-mêmes de deux types: soit il s'agit de polluants dont l'effet est

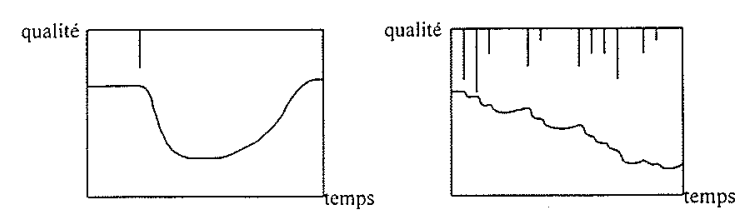

1. Effets de choc et effets cumulatifs. durable, comme les métaux lourds ou certains micropolluants toxiques, soit cet impact est dû à l'accumulation dans les sédiments de polluants qui sont progressivement relargués dans l'eau. Dans les deux cas, l'étude de ces impacts passe par une évaluation des apports totaux des rejets dans le milieu sur de longues périodes. L'année est généralement prise comme période de référence.

Les effets chroniques sur la biocénose sont également très souvent mentionnés. Ils sont caractérisés par la nonrécupération des populations animales et végétales entre deux dégradations successives de la qualité de l'eau, et donc, comme nous le représentons dans la figure 2, par une altération progressive de ces populations (dans la quantité ou la diversité) due à la répétition des chocs de pollution. Le facteur susceptible d'influencer ce type de phénomène est donc la fréquence de retour des événements perturbateurs. Certains auteurs avancent même l'idée qu'une même masse de pollution introduite dans le milieu sous une forme discontinue serait plus préjudiciable pour ce dernier que si elle est introduite de façon continue (notion d'effet de stress). Cependant, aucune démarche expérimentale n'a permis de démontrer ce phénomène qui est perçu de manière intuitive [Chocat et al., 1993].

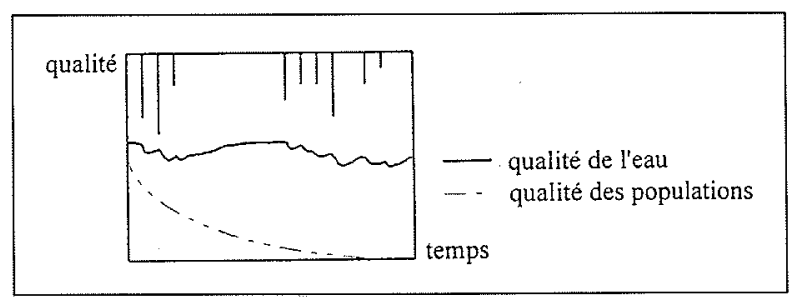

2. Représentation possible des effets chroniques.

Il est donc nécessaire de prendre en compte différentes échelles de temps et d'espace en fonction des rejets considérés, mais aussi en fonction des polluants, comme le montrent les deux graphiques suivants, proposés par [Driscoll, Mancini, 1979], complétés par [Aalderink, Lijklema, 1985] et extraits de [Trabuc, 1989]:

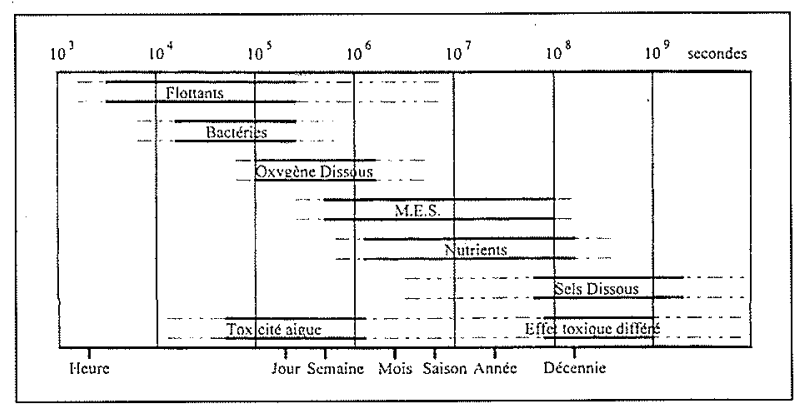

3. Echelles de temps relatives à l'impact des rejets urbains. 


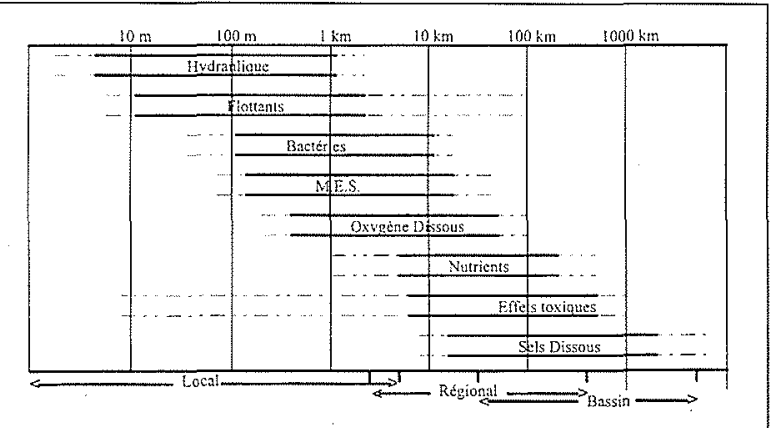

4. Echelles d'espace relatives à l'impact des rejets urbains.

\subsection{Les différents phénomènes d'impact}

Lors d'un rejet, il se produit une cascade d'événements qui vont chacun à leur niveau perturber l'écosystème. Nous proposons de distinguer les indicateurs d'impact relatifs aux phénomènes en jeu, en les hiérarchisant sur trois niveaux :

- Les impacts physico-chimiques, qui ne font intervenir que le paramètre de pollution considéré indépendamment des autres polluants. Ce premier niveau, défini comme étant l'effet par [Chocat et al., 1993] est la conséquence directe et immédiate de l'apport de polluants dans le milieu, par exemple l'augmentation de la concentration d'un produit donné dans l'eau.

- Le second niveau, que nous intitulerons impact biochimique, regroupe l'ensemble des réactions biochimiques qui se produisent dans le biotope: autoépuration, relargage, consommation d'oxygène due à la dégradation de la matière organique, etc.

- Enfin, le troisième niveau est l'impact biologique. Il peut être relatif soit aux conséquences directes des rejets sur la biocénose, soit aux conséquences en cascade provoquées par les impacts des deux niveaux précédents.

Le tableau suivant synthétise l'ensemble des phénomènes d'impact, selon ces trois niveaux, en distinguant de plus les effets à court et à long terme. La classification proposée est très simplifiée. Il y a en effet de nombreuses boucles de rétroaction entre biotope et biocénose, et les cycles biochimiques sont beaucoup plus complexes que la description que nous en faisons ici. Cependant, cette classification permet d'organiser la caractérisation des différents phénomènes d'impact.

Comme nous l'avons indiqué précédemment ces différents impacts peuvent être considérés selon deux perceptions différentes :

- La perception écologique de l'impact, relative à la qualité du milieu naturel, qui regroupe l'ensemble des impacts susceptibles d'altérer l'écosystème.

- La perception environnementaliste de l'impact, c'està-dire les phénomènes susceptibles de perturber, voire de rendre impossibles les usages souhaités du milieu aquatique.

Les deux paragraphes suivants détaillent les différents impacts selon ces deux points de vue.

\subsection{Les impacts sur l'écosystème}

1.3.1 La sous-oxygénation des milieux et les chocs anoxiques

Les milieux qui ont tendance à être sous-oxygénés sont soit des eaux relativement chaudes, soit des eaux profondes, dans lesquelles la stratification thermique peut être res. ponsable d'une sous-oxygénation des couches inférieures, soit encore des milieux peu brassés. L'apport continu de quantités importantes de matières organiques contribue à maintenir le milieu à un taux d'oxygène dissous relativement faible. Ces milieux sont particulièrement sensibles aux désoxygénations brutales provoquées par les rejets urbains de temps de pluie. Ce phénomène est susceptible de produire des mortalités piscicoles importantes. A titre d'exemple [Chebbo et al., 1995] notent que dans l'agglomération parisienne, des mortalités piscicoles consécutives à des orages adviennent tous les deux à trois ans.

\subsubsection{Les impacts dus aux apports de micropolluants}

Les micropolluants, qu'ils soient organiques ou minéraux ont les particularités suivantes:

- L'activité des produits dépend de leur forme physicochimique qui est elle-même fonction de conditions extérieures.

- Les micropolluants sont souvent stockés dans les sédiments et relargués de façon lente.

- Ils ont une rémanence et une influence spatiale très grandes.

- Ils peuvent être toxiques à de très faibles concentrations.

Les rejets urbains de temps de pluie sont particulièrement mis en cause pour les apports en métaux lourds (plomb, zinc, cuivre et parfois cadmium).

\subsubsection{Les impacts hydrauliques et morphodynamiques}

Les rejets urbains de temps de pluie ont un impact hydraulique et morphodynamique parfois notable sur les cours d'eau, de par l'importance des débits pouvant être déversés. Cet impact est d'autant plus fort qu'il s'agit de petits cours d'eau ou de rivières sujettes à de forts étiages. Du point de vue de la qualité de l'eau, il est responsable de la remise en suspension des sédiments et done de la disponibilité des polluants accumulés. Un autre impact du même type est l'impact morphodynamique : l'apport important de matières en suspension peut engendrer un envasement du lit et donc une modification de l'écoulement entraînant des zones de ralentissement ou d'accélération hydraulique.

\subsubsection{L'eutrophisation}

L'hyper-eutrophisation des milieux, considérée initialement comme un problème spécifiçue aux plans d'eau, s'avère être également un problème préoccupant pour certains cours d'eau et zones littorales. La contribution des rejets urbains de temps de pluie à ce type d'impact est 


\begin{tabular}{|c|c|c|}
\hline $\begin{array}{c}\text { Niveau } \\
\text { d'impact }\end{array}$ & Impact immédiat & Impact cumulatif ou différé \\
\hline $\begin{array}{l}\text { Niveau } 1 \\
\text { Impacts } \\
\text { physico-chimiques }\end{array}$ & $\begin{array}{l}\text { Augmentation de la concentration dans l'eau et } \\
\text { dans les sédiments: } \\
\text { - turbidité (M.E.S.), } \\
\text { - matière organique et nutriments, } \\
\text { - bactéries pathogènes, } \\
\text { - micropolluants dissous ou facilement relar- } \\
\text { gables, }\end{array}$ & $\begin{array}{l}\text { - Envasement et colmatage (M.E.S.). } \\
\text { - Accumulation dans les sédiments : matière } \\
\text { organique et nutriments; polluants persis- } \\
\text { tants: métaux lourds, hydrocarbures et tous } \\
\text { les micropolluants. } \\
\text { - Relargage, remise en suspension et trans- } \\
\text { port de polluants (crues ou gros événements } \\
\text { pluvieux) (physique). } \\
\text { - Evolution des polluants }+/ \text {-disponibles ou } \\
\text { actifs (chimique). }\end{array}$ \\
\hline $\begin{array}{l}\text { Niveau } 2 \\
\text { Impacts } \\
\text { biochimiques }\end{array}$ & $\begin{array}{l}\text { - Diminution du taux d'oxygène : } \\
\text { - films d'hydrocarbures à l'interface air-eau, } \\
\text { - dégradation de la matière organique. } \\
\text { - Transformation azote ammoniacal en ammo- } \\
\text { niac non ionisé (toxique), si le pH est élevé. } \\
\text { - Développement de bactéries pathogènes en } \\
\text { certains points favorables du milieu. }\end{array}$ & $\begin{array}{l}\text { - Consommation d'oxygène dissous due à la } \\
\text { dégradation de la matière organique progres- } \\
\text { sivement relarguée et rendue disponible. }\end{array}$ \\
\hline Niveau 3 & Impacts biologiques & \\
\hline $\begin{array}{l}\text { Sur la flore } \\
\text { aquatique }\end{array}$ & $\begin{array}{l}\text { (La chute de luminosité et l'augmentation de la } \\
\text { turbidité au cours d'un événement pluvieux sont } \\
\text { responsables, entre autres, d'une chute de l'ac- } \\
\text { tivité photosynthétique, et donc probablement } \\
\text { du taux d'oxygène dissous.) }\end{array}$ & $\begin{array}{l}\text { - Déséquilibre de la croissance phytoplancto- } \\
\text { nique : hyper-eutrophisation et/ou disparition } \\
\text { de certaines especes par apport de nutriments } \\
\text { (du facteur limitant } N \text { ou P), directement dis- } \\
\text { sous ou par relargage. }\end{array}$ \\
\hline Sur la faune & $\begin{array}{l}\text { Mortalité de la faune aquatique, notamment pis- } \\
\text { cicole, lors de chocs de pollution: } \\
\text { - Soit par asphyxie, par manque d'oxygène dis- } \\
\text { sous (M.O.) ou par colmatage des ouies } \\
\text { (M.E.S.). } \\
\text { Soit par toxicité aiguë (généralement de } \\
\text { source industrielle). }\end{array}$ & $\begin{array}{l}\text { - Introduction des toxiques des sédiments } \\
\text { dans la chaîne alimentaire par les vers. } \\
\text { - Concentration dans certains organismes. } \\
\text { - Mutation des populations animales, raréfac- } \\
\text { tion des plus sensibles. }\end{array}$ \\
\hline
\end{tabular}

Tableau 1. - Classification des phénomènes d'impact selon les trois niveaux proposés et la durée.

probablement relativement faible. Cette altération du milieu doit cependant être considérée car elle augmente le risque de désoxygénation.

\subsection{Les impacts environnementaux}

La préservation de la qualité de l'environnement dépasse largement la défense désintéressée de milieux ou d'espèces menacées. La dégradation des milieux a en effet un impact économique non négligeable par la perturbation des activités qu'elle peut engendrer. Comme [Chocat et al., 1993], nous avons regroupé ces usages en trois catégories: le captage d'eau, notamment pour la fabrication d'eau potable, la pisciculture et la conchyliculture, les activités de loisir.

Le captage d'eau concerne l'abreuvement, l'irrigation, l'industrie et la fabrication d'eau potable. Les deux derniers usages sont les plus contraignants en ce qui concerne la qualité physico-chimique de l'eau. Les contraintes pour la production d'eau potable sont strictement réglementées et doivent être respectées $100 \%$ du temps. Les rejets urbains de temps de pluie perturbent fréquemment les captages en rivière, en particulier du fait de leur forte charge en matières en suspension qui colmatent les filtres. La production d'eau potable peut également être perturbée par la présence de nitrates, de produits phytosanitaires, d'hydrocarbures et par le développement de certaines algues (algues bleues).
La pisciculture et la conchyliculture sont également deux activités très sensibles aux dégradations de la qualité du milieu. La pisciculture en eau douce est généralement pratiquée dans des zones relativement préservées, dans un milieu bien oxygéné et rarement à l'aval de rejets urbains importants. La conchyliculture semble par contre plus exposée et plus sensible à la pollution urbaine de temps de pluie qui entraîne des rejets importants de bactéries pathogènes. Les coquillages, en filtrant de grandes quantités d'eau, se comportent en effet comme de véritables éponges à polluants. Cette accumulation dans la chair des coquillages peut provoquer l'intoxication d'un nombre éventuellement important de personnes et des pertes d'exploitation pour les conchyliculteurs.

Les pollutions visuelles (la présence de ffottants, de laisses de crues, d'irisations, une turbidité ou une eutrophisation excessives) et les pollutions olfactives constituent des impacts forts pour différentes activités de loisir : la promenade, les jeux d'eau, la baignade, la pêche et sur les côtes le ramassage des coquillages et la pêche à pied.

Les lieux de baignade sont essentiellement sensibles à la qualité bactériologique de l'eau. Ces espaces doivent également être préservés d'une trop forte turbidité et d'une eutrophisation excessive. La figure 5, issue d'une enquête effectuée à la demande du ministère de l'environnement, met en évidence le rôle des rejets de temps de pluie comme cause de pollution des baignades en mer [Courant, 1994]. 


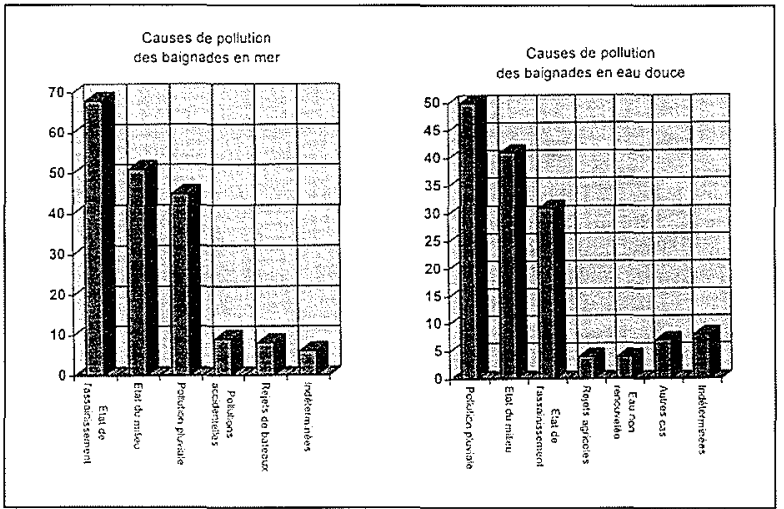

5. Causes identifiées de pollution des baignages en mer ( 164 cas étudiés) et en eaux douces (228 cas étudiés) en pourcentage de réponses reçues.

La pêche est une activité de loisir très révélatrice de l'état de l'écosystème. La qualité des poissons présents constitue un bon critère intégrateur de la qualité du milieu. La vie piscicole est notamment sensible aux chocs anoxiques, à une trop forte turbidité et à la présence de toxiques dont l'ammoniac non ionisé, altérations qui sont toutes liées aux rejets urbains de temps de pluie. La pratique de la pêche à pied nécessite les mêmes précautions que la conchyliculture.

On peut imaginer que l'impact des rejets sur l'ensemble de ces activités de loisirs ait des répercussions économiques non négligeables par la baisse de fréquentation des sites touristiques. Si aucune étude précise ne permet de valider cette supposition, la médiatisation et les moyens d'information sur la qualité de l'eau mis à la disposition des vacanciers laissent présager une évolution vers une plus grande exigence de leur part.

\section{LA VULNÉRABILITÉ PARTICULIÈRE DES DIFFÉRENTS HYDROSYSTÈMES NATURELS}

L'analyse de l'impact des rejets urbains doit être conduite en fonction de la nature du milieu récepteur : cours d'eau, plans d'eau, nappes souterraines, milieux estuariens, côtiers ou marins. Les phénomènes de pollution dépendent de la nature du milieu, de son fonctionnement hydrodynamique, de son état de dégradation, de sa capacité d'autoépuration, des formes de vie dont il est le siège, de ses usages et de son environnement. «Les impacts les plus préoccupants et les rejets les plus dégradants ne seront pas les mêmes selon qu'il s'agit du Rhône ou de la Seine, en mars ou en octobre, d'un lac profond ou d'une lagune, d'une nappe superficielle ou profonde, du littoral avec des plages à proximité, dans une baie ou sur un grand courant, etc. 》 [Tabuchi, Bachoc, 1993].

\subsection{Le milieu littoral}

La sensibilité du milieu littoral dépend essentiellement de ses caractéristiques hydrodynamiques, qui sont très diverses selon le point considéré. Le milieu littoral est notamment sensible:
- aux apports de matières en suspension qui, en augmentant la turbidité de l'eau, peuvent ralentir l'activité photosynthétique, premier maillon de la chaîne alimentaire,

- aux apports de sels nutritifs, causant un emballement du cycle trophique pouvant aller jusqu'à l'asphyxie du milieu.

- et aux apports de micropolluants, qui ont la particularité d’être immédiatement toxiques pour la faune et la flore à de très faibles doses et de s'accumuler dans la chaine alimentaire.

Les usages pratiqués en milieu littoral (baignade, thalassothérapie, pêche à pied, conchyliculture, etc.) rendent également ce milieu fortement sensible à la pollution bactériologique.

\subsection{Les plans d'eau}

Les plans d'eau sont particulièrement touchés par les problèmes d'hyper-eutrophisation si les rejets de nutriments sont importants. Ils ne sont pas non plus à l'abri des chocs anoxiques puisque les eaux sont peu brassées. Selon les usages, comme le milieu marin, ils peuvent nécessiter une protection sanitaire importante.

\subsection{Les eaux souterraines}

Les eaux souterraines sont également affectées par la pollution générée par l'activité humaine. Cette pollution est d'autant plus inquiétante que les nappes constituent une source importante d'eau potable. Les pollutions accidentelles ont constitué la première préoccupation en ce qui concerne la protection des nappes. Celles-ci sont également touchées par des pollutions diffuses et chroniques, notamment par l'infiltration des eaux de ruissellement qui lessivent les surfaces urbaines et agricoles. Les indicateurs pris en compte ont d'abord été les éléments conservatifs (métaux lourds), puis les polluants organiques hydrophobes et enfin la pollution bactérienne, le plus souvent d'origine agricole (engrais, fumures d'élevages intensifs, produits phytosanitaires, etc.) [Ledoux, 1993] mais qui peut également avoir une origine urbaine.

\subsection{Les cours d'eau}

Les cours d'eau sont non seulement des milieux sensibles aux pollutions, mais ils sont également le vecteur de la pollution générée par les bassins urbains qui les bordent, vers des milieux éventuellement plus sensibles comme les plans d'eau ou des zones littorales où sont pratiquées la pêche à pied, la conchyliculture et la baignade. Il est donc nécessaire de tenir compte de la sensibilité des milieux dans lesquels les cours d'eau sont susceptibles de se déverser afin d'assurer la cohérence de la politique de préservation de la qualité des milieux aquatiques.

La hiérarchisation des problèmes d'impact est également difficile d'un point de vue théorique pour les cours d'eau, car ces derniers peuvent être touchés par toutes les formes de pollution. L'identification des impacts doit s'appuyer sur l'observation et la connaissance du milieu étudié et de son enviromnement. Une revue historique des problèmes constatés peut être un bon indicateur de la sensibilité du 
milieu. A défaut, il est possible de raisonner a priori, à partir des caractéristiques physiques du milieu. On peut par exemple distinguer les cours d'eau qui ont un étiage soutenu et ceux qui ont un étiage faible. Ces derniers seront particulièrement vulnérables aux rejets urbains de temps de pluie en période d'étiage, générateurs de chocs anoxiques et de mortalités piscicoles. La vitesse peut également être un critere de distinction. En effet, une vitesse lente sera favorable au développement phytoplanctonique et le milieu sera donc plus sensible aux apports de nutriments. Les petits cours d'eau constituent une palette de situations très diversifiées: les torrents de montagne, généralement bien oxygénés mais avec de forts débits d'étiage; les petits cours d'eau urbains, couverts ou canalisés, servant d'égout naturel ; les cours d'eau réintroduits dans le paysage urbain afin de constituer des lieux de promenade, particulièrement sensibles à la pollution visuelle et olfactive.

\section{CONCLUSIONS}

Les objectifs de réduction des rejets doivent maintenant être définis de façon à atteindre des objectifs de qualité des milieux. Ceci impose de disposer de méthodes et d'outils afin de quantifier l'influence d'une réduction des rejets sur l'évolution de la qualité du milieu, c'est-à-dire permettant :

- de mesurer l'impact actuel des rejets,

- de simuler les conséquences sur le milieu, d'une action sur le système d'assainissement.

Les phénomènes d'impact liés aux rejets urbains de temps de pluie sur les milieux naturels sont maintenant correctement identifiés. Cependant leur quantification, nécessaire pour mener à bien les études, reste délicate essentiellement pour trois raisons:

- complexité des interactions entre les différents phénomènes, qui rend difficile l'identification des indicateurs les plus pertinents ;

- caractère événementiel des rejets, qui complique toute tentative d'acquisition de données sur le site;

- absence d'outil générique permettant de relier les rejets aux impacts.

L'application des nouveaux textes réglementaires risque donc de soulever de nombreuses difficultés de terrain. Un des pièges principaux à éviter est que la recherche d'un trop grand pragmatisme amène à utiliser des critères ou des méthodes trop schématiques pour tenir compte de la complexité réelle du système étudié. Il est certain que les rails étant maintenant posés, il est indispensable de faire démarrer le train. Méfions-nous cependant de ne pas vouloir aller trop vite, et prenons la précaution d'observer en permanence les conséquences de nos actions de façon à pouvoir changer de direction si cela s'avère nécessaire.

\section{Références}

AALDERINK R.H., LIJKLEMA L. (1985). - Water quality effects in surface waters receiving storm water discharges. Water in urban areas. TNO Commintee on Hydrological Research, Proceedings and Informations $n^{\circ} 33$, The Hague, TNO, pp. 143-159.

Bachoc A., Tabuchi J.P., Chebbo G., Phillppe J.P. (1993). La pollution des rejets urbains par temps de pluie : quan- tité, origine et nature. In "La plaie, source de vie, choc de pollution \%. S.H.F. Paris. 17 ef 18 mars 1993. La Houlle Blanche, $\mathrm{n}^{\circ} 1 / 21994$, pp. 21-33

BARroIN G. (1991) - La réluabilitation des plans d'eau. La Recherche. n²38. Déc. 1991. Vol. 22, pp. 1412-1422.

Chebbo G., Mouchel J.M., Saget A., Gouzallles M. (1995) - La pollution des rejets urbains par temps de pluie : flux, nature et impacts in numéro spécial TSM à parâ̂te 1995.

Chocat B., Cathelain M., Mares A., Mouchel J.M. (1993) - La pollution due aux rejets urbains de temps de pluie Impacts sur les milicux récepteurs. Exposé introductif. In «La pluie, source de vie, choc de pollution». S.H.F. Paris 17 et 18 mars 1993. La Houlle Blanche $\mathrm{n}^{\circ} 1 / 21994$, pp. 97-105.

CHOCAT B. et al. (à paraître en 1996)-Association eurydice 92 : Encyclopédie de l'hydrologie urbaine et de l'assainissement ; Ed. Tec et Doc-Lavoisier; Paris ; à paraîre 1995.

Courant (1994). - Le meaudec: le marché de l'eau et des déchets. Numéro hors-série, Courant, Paris.

Directive Européenne (1991): Directive du conseil du 21 mai 1991 relative au traitement des eaux résiduaires urbaines (91/271/CEE). Journal Officiel des Communautés Européennes du 30.05.97, No L 135/40 à 52 .

DRISCOLL M., MANCINI M. (1979) - Benefits analysis for combined sewer overflow control. Cincinnati : U.S.E.P.A. (Environinemental Policy Agency), avril 1979, 48 pages.

FÉRA Ph. (1993) - Assainissement en zone littorale touristique. 1 - Sensibilité du milieu marin littoral aux apports tellum riques. In TSM $n^{\circ}$ 3/1993. Spécial Assainissement, mars 1993, pp. 105-110.

Herremans L., Zobrist C., Chaumontet M. (1993). - Eude du transfert de pollution par temps de pluie sur le réseau d'assainissement du District Urbain d'Arras. "La plaie, source de vie, choc de pollution". S.H.F. Paris 17 et 18 mars 1993. La Houlle Blanche, n० 1/2 1994, pp. 7579

LE Molgne J.L. (1984), — La théorie du système général. Presses Universitaires de France. Paris. Seconde Edition, 215 pages.

LEDOux E. (1993) - Modélisation du transport des polluants par les eaux souterraines. Séminaire de programmation « $\mathrm{Mo}$ délisation du comportement des polluants dans les hydrosystènes ». Ministère de l'Environnement. Partis, mai 1993, 11 pages.

Ministère de l'Environnement (1991). - Assainissement des collectivités locales. Etat de l'équipement et des financements ; Ministère de l'environnement.

Péricou V., Martaud M., Teillot J.P., Vandevenne M. (1992) - Maîtrise de limpact des rejets de temps de pluie des réseaux unitaires d’agglomérations de l'Est de la France : Longwy, Metz, Mulhouse. Novatech, GRAIE, Lyon, novembre 1992, pp. 223-234.

TABUCHI J.P., BACHOC A. (1993) - Proposition d'un programme national de recherche et d'expérimentation sur la pollution des rejets urbains de temps de pluie. Groupe de Travail AGHTM : "Les rejers urbains de tenus de pluie ". Paris, avril 1993,19 pages.

Trabuc P. (1989) - Prise en compte de l'effet polluant des rejets urbains de temps de pluie. Recommandations pour l'élaboration ou la révision d'un schéma d'assainissement. Agence de Bassin Seine-Normandie. Décembre 1989, 45 pages.

WOLFF E. ep. Brelot (1994). - Eléments pour la prise en compte de l'impact des rejets urbains sur les milieux naturels dans la gestion des systèmes d'assainissement. Thèse, INSA, Laboratoire méthodes, Villeurbanne, novembre 1994,320 pages. 